\title{
Neutron skin studies in heavy nuclei with coherent $\pi^{0}$ photo-production
}

\author{
Maria Isabel FERRETTI BONDY*a, on behalf of the A2 Collaboration \\ ${ }^{a}$ Institut für Kernphysik, Johannes Gutenberg Universität Mainz, \\ Johann-Joachim-Becher-Weg 45, 55128 Mainz, Germany \\ E-mail: ferretti@kph.uni-mainz.de
}

The charge distribution of nuclei is known with very high accuracy, i.e. in electron scattering experiments, conversely, the mass distribution is experimentally less accessible and therefore less precisely known. An accurate determination of the neutron density distribution is of particular interest. Especially in nuclei with $\mathrm{N} » \mathrm{Z}$, a strong neutron skin is expected, since the excess neutrons are pushed outwards against surface tension by the Coulomb forces. With the precise experimental determination of the neutron skin thickness essential constraints for the nuclear equation of state (EOS) can be provided and thus allowing to draw conclusions on the size of neutron stars.

The method of coherent $\pi^{0}$ photoproduction, $A\left(\gamma, \pi^{0}\right) A$, provides a powerful tool to determine the mass distribution of various nuclei. In a novel experimental campaign carried out in 2012 within the A2 collaboration at the Mainz Microtron (MAMI), five nuclei have been measured: ${ }^{58} \mathrm{Ni},{ }^{116,120,124} \mathrm{Sn},{ }^{208} \mathrm{~Pb}$. The tin targets are of special interest because most of the systematic errors due to pion-nucleon interaction can be neglected, and they allow a precise investigation along an isotopic chain.

First results of these studies will be discussed.

53rd International Winter Meeting on Nuclear Physics,

26-30 January 2015

Bormio, Italy

\footnotetext{
*Speaker.
} 


\section{Introduction}

The neutron skin thickness $\Delta R_{n p}$ is given by the difference between the Root Mean Square (RMS) radii of the neutron and proton distributions:

$$
\Delta R_{n p}=\left\langle r_{n}^{2}\right\rangle^{\frac{1}{2}}-\left\langle r_{p}^{2}\right\rangle^{\frac{1}{2}}
$$

The size and shape of atomic nuclei has been extensively studied in nuclear physics [1,2]. As a result, the charge distribution in nuclei is very accurately determined. In contrast, the measurement of the neutron distribution has proven to be more difficult to access. Different experiments have tried to address this open question by scattering particles such as protons [3], pions [4] or antiprotons [5] from nuclei. However, because of uncertainties in the strong interaction [6], the interpretation of these results is model dependent.

By measuring the neutron skin, a better understanding about finite nuclei as well as about objects in astrophysics like neutron stars can be obtained. The connection is given by the Equation of State (EOS) of nuclear matter, that can be expressed as

$$
E(\rho, \delta)=E(\rho, 0)+E_{s y m}(\rho) \delta^{2}+O\left(\delta^{4}\right)
$$

where $\rho=\rho_{n}+\rho_{p}$ is the total nucleon density and $\delta=\frac{\rho_{n}-\rho_{p}}{\rho}$ the relative asymmetry. $E(\rho, \delta)$ is the energy per particle in asymmetric nuclear matter and $E_{s y m}(\rho)$ the symmetry energy. Expanding the EOS around saturation density, the symmetry energy $E_{\text {sym }}(\rho)$ can be written as:

$$
E_{\text {sym }}(\rho) \approx J-L\left(\frac{\rho_{0}-\rho}{3 \rho_{0}}\right)+\frac{1}{2} K_{s y m}\left(\frac{\rho_{0}-\rho}{3 \rho_{0}}\right)^{2} .
$$

The symmetry energy coefficient $J=E_{\text {sym }}\left(\rho_{0}\right)$ has been experimentally constrained between 30 and $35 \mathrm{MeV}[7,8]$ and can be successfully predicted by nuclear mean-field models. On the other hand, for the density dependence of the symmetry energy given by the slope parameter $L$ and the curvature parameter $K_{\text {sym }}$ the discrepancy between experimental values and various model predictions is much larger $[9,10]$.

Measuring the neutron skin thickness of heavy nuclei can help to constrain the slope parameter $L$. Mean-field models that reproduce the binding energy of heavy nuclei like ${ }^{208} \mathrm{~Pb}$, also result in a linear correlation between the neutron skin thickness and the slope parameter $L$ of the symmetry energy at saturation density, calculated with the same model [11-13](see figure 1). 


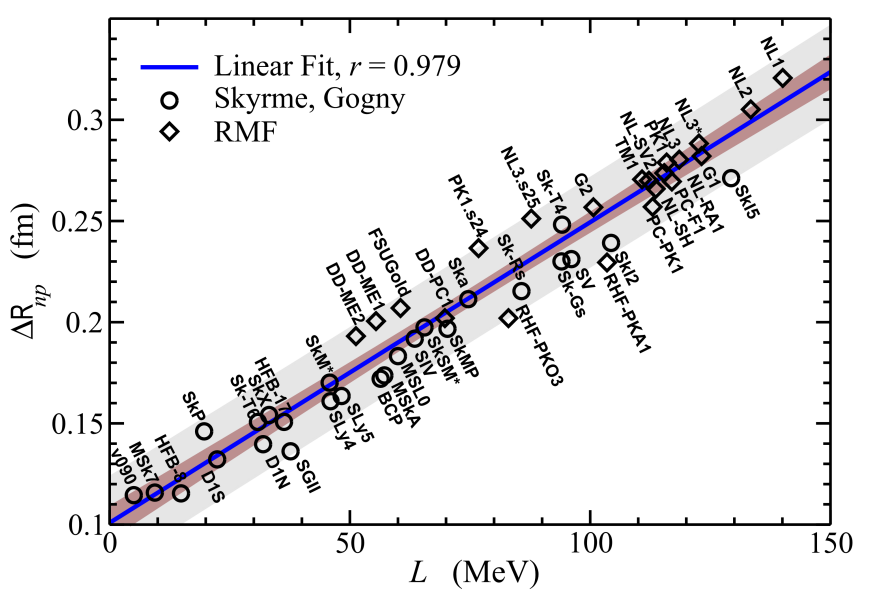

Figure 1: Neutron skin thickness $\Delta R_{n p}$ of ${ }^{208} \mathrm{~Pb}$ as a function of the slope parameter L of the symmetry energy at saturation density [14]. The linear correlation given from different theoretical predictions is shown.

\section{Coherent $\pi^{0}$ photoproduction and experimental setup}

One powerful method to measure the neutron distribution using electromagnetic probes is coherent $\pi^{0}$ photoproduction [15-18]. Here, a photon beam impinges on a nucleus with a mass number A, probing the entire volume. In the final state the nucleus stays in its ground state and the produced $\pi^{0}$ will decay into two photons:

$$
\gamma+A_{(g s)} \longrightarrow A_{(g s)}+\pi^{0}
$$

The protons and neutrons of the target material contribute equally to the total cross section for the coherent $\pi^{0}$ photoproduction, resulting in a sensitivity to the matter distribution of the nucleus [17].

In the simplest theoretical description, the plane wave impulse approximation (PWIA), the differential cross section for the coherent $\pi^{0}$ photoproduction for nuclei with spin zero can be written as:

$$
\frac{d \sigma_{P W I A}}{d \Omega}\left(E_{\gamma}, \Theta_{\pi}\right)=\frac{s}{m_{N}^{2}} A^{2} \frac{d \sigma_{N S}}{d \Omega^{*}}\left(E_{\gamma}^{*}, \Theta_{\pi}^{*}\right) F^{2}(q)
$$

with

$$
\frac{d \sigma_{N S}}{d \Omega^{*}}\left(E_{\gamma}^{*}, \Theta_{\pi}^{*}\right)=\frac{1}{2} \frac{q_{\pi}^{*}}{k^{*}}\left|F_{2}\left(E_{\gamma}^{*}, \Theta_{\pi}^{*}\right)\right|^{2} \sin ^{2}\left(\Theta_{\pi}^{*}\right) .
$$

$E_{\gamma}$ is the incident photon energy and $\Theta_{\pi}$ the pion polar angle, both in the photon-nucleus center of mass system $(\mathrm{CM}) . m_{N}$ is the nucleon mass, $q\left(E_{\gamma}, \theta_{\pi}\right)$ the momentum transfer to the nucleus and $F(q)$ is the nuclear matter form factor. $\sqrt{s}$ is the total energy of the photon-nucleon pair, $E_{\gamma}^{*}, k^{*}$ are the photon energy and the momentum, respectively, both in the photon-nucleon $\mathrm{CM}, \Theta_{\pi}^{*}, q_{\pi}^{*}$ are the pion angle and momentum, respectively, also in the photon-nucleon CM [17].

In the PWIA all the final state interactions (FSI) between the $\pi^{0}$ and the nucleus are neglected such, that the $\pi^{0}$ leaves the nucleus as a plane wave. Nevertheless, the sensitivity of the differential cross section to the matter distribution given by the square of the nuclear matter form factor $F(q)$ (see Eq. 2.2) is visible. 
By using an electromagnetic probe, no initial state interactions have to be taken into consideration for the interpretation. However, for the full theoretical description, the FSI between the $\pi^{0}$ and the nucleus have to be accounted for [19].

The experiment was carried out within the A2 collaboration in Mainz at the Mainz Microtron (MAMI) [20]. MAMI is a continues wave electron accelerator. The electron beam can be polarized or unpolarized, reaching energies up to $1600 \mathrm{MeV}$ and currents up to $100 \mu \mathrm{A}$.

To produce an energy marked photon beam, the unpolarized electron beam with an energy of $E_{0}=855 \mathrm{MeV}$ hits a copper radiator $(10 \mu \mathrm{m}$ thick). The recoiled electrons are deflected in the magnet of the Glasgow photon tagger [21,22] where their energy $E_{e^{-}}$is measured in the focal plane detectors with a resolution of $\approx 2 \mathrm{MeV}$ FWHM. In this way, the energy of the photons can be calculated as $E_{\gamma}=E_{0}-E_{e^{-}}$. The photon beam is collimated and directed towards the target which is surrounded by the detector system.

The isotopically enriched targets used in this experimental campaign were three tin isotopes $\left({ }^{116,120,124} \mathrm{Sn}\right)$, one nickel target $\left({ }^{58} \mathrm{Ni}\right)$ and a lead target $\left({ }^{208} \mathrm{~Pb}\right)$, each with a purity higher than $99.5 \%$.

The main components of the detector system are the Crystal Ball (CB) [23] and the TAPS $[24,25]$ calorimeter. The $\mathrm{CB}$ detector, consisting of $672 \mathrm{NaI}$ detector elements, and the TAPS detector, made of $366 \mathrm{BaF}_{2}$ and $72 \mathrm{PbWO}_{4}$ elements, cover together $97 \%$ of the full solid angle. This, together with the high energy resolution, makes the combined system of CB and TAPS well suited to detect multiphoton final states.

In addition there are three inner detectors for charged particle identification located around the beamline in the center of the CB: one Particle Identification Detector (PID) and two multiwire proportional chambers (MWPCs). The PID consist of 24 plastic scintillators arranged in the form of a cylinder parallel to the beam direction. Between the PID and the CB, two MWPCs are located. The MWPCs where used to determine the target position relative to the center of the CB.

\section{Analysis and Preliminary Results}

The $\pi^{0}$ 's in the final state were identified via their $2 \gamma$ decay by reconstructing the invariant mass. From the energy and angle information of the detected photons, the momentum of the $\pi^{0}$ was deduced. In order to select the coherent events, the missing energy $\Delta E_{\pi}^{\text {Miss }}$ was calculated and plotted as a function of the momentum transfer q (see Fig: 2):

$$
\Delta E_{\pi}^{M i s s}=E_{\pi, D e t}^{C M}\left(\gamma_{1} \gamma_{2}\right)-E_{\pi, T h}^{C M}\left(E_{\gamma}\right) .
$$

$E_{\pi, D e t}^{C M}\left(\gamma_{1} \gamma_{2}\right)$ is the pion energy in the CM reconstructed from the two measured photons $\gamma_{1}$ and $\gamma_{2}$. $E_{\pi, T h}^{C M}\left(E_{\gamma}\right)$ is the expected pion energy in the CM that a pion produced in a photoreaction should have, if the energy of the incoming photon was $E_{\gamma}$.

This was done for all five targets in the energy range from $150 \mathrm{MeV}$ up to $300 \mathrm{MeV}$, using 10 $\mathrm{MeV}$ steps. The events corresponding to the coherent reaction should be centered around $\Delta E_{\pi}^{M i s s}=$ 0 . On the other hand, the incoherent events are shifted to negative values of the missing energy. This can be used to separate coherent from incoherent events.

To perform this separation, the projection of every single momentum transfer-bin (bin size $\Delta q$ $=0.01 \mathrm{fm}^{-1}$ ) was done and a double Gaussian was fitted. One of the Gaussian corresponds to the 

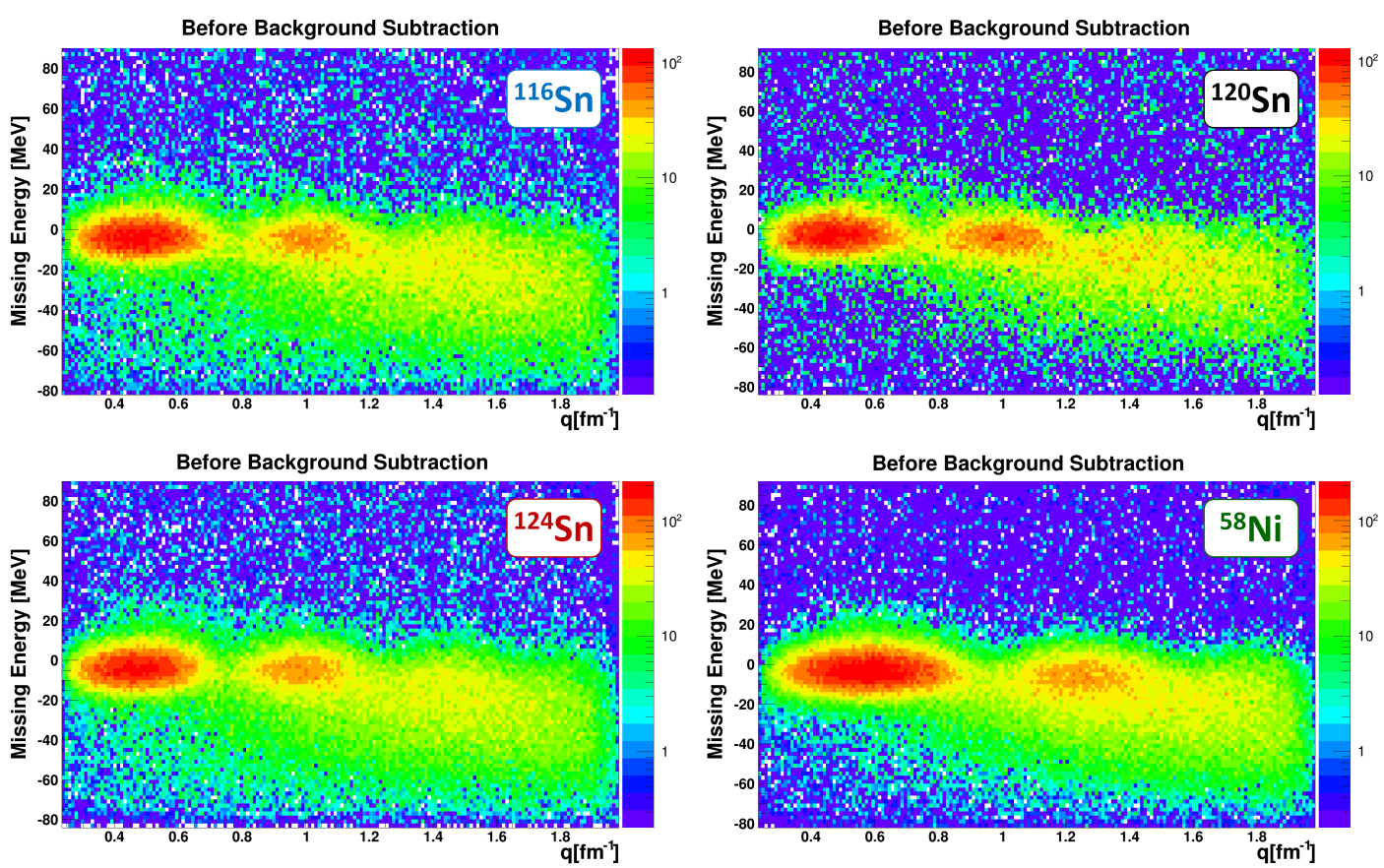

Figure 2: Missing energy as a function of the momentum transfer before background subtraction for ${ }^{116,120,124} \mathrm{Sn}$ and ${ }^{58} \mathrm{Ni}$. Here, the photon energy range was $E_{\gamma}=210$ to $220 \mathrm{MeV}$.

coherent peak, the other one to the incoherent background (see Figure 3). The position and width of the coherent peak was estimated in the first maxima region where the incoherent contribution is expected to be negligible and hold constant for the whole momentum transfer range q. Once the background contribution was estimated, the incoherent events could be subtracted. The result of this procedure for incoming photons in the energy range $E_{\gamma}=210$ to $220 \mathrm{MeV}$ for the three tin isotopes and the nickel target can be seen in the figure 4 .
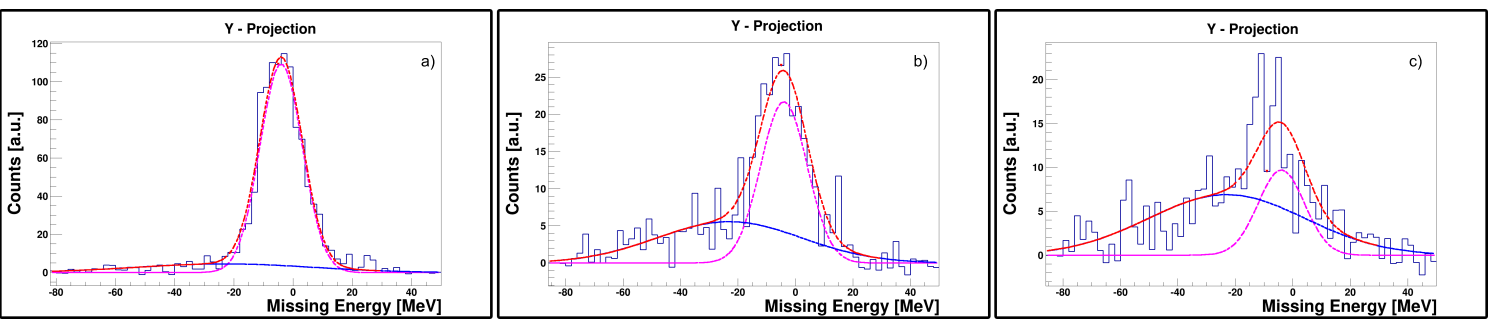

Figure 3: Fit procedure to estimate the incoherent background contribution, here for ${ }^{116} \mathrm{Sn}$ in the photon energy range $E_{\gamma}=210$ to $220 \mathrm{MeV}$. The magenta curve corresponds to the coherent contribution, the blue curve to the incoherent background, the red curve is the sum of both.

Projection of one q-bin a): in the first maxima region, b): between the first maxima and the first minima region, c): in the first minima region. 

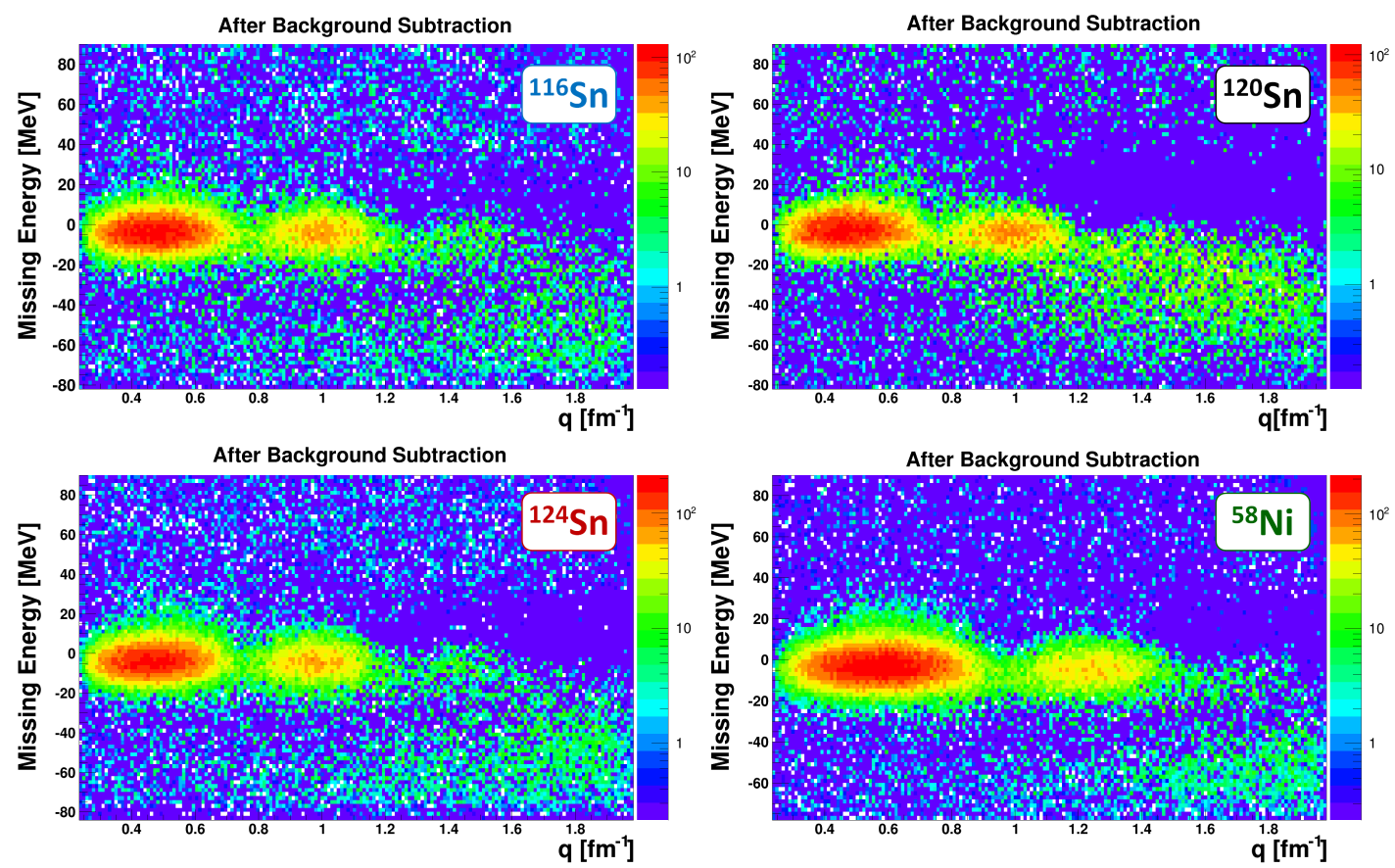

Figure 4: Missing energy as a function of momentum transfer after background subtraction for ${ }^{116,120,124} \mathrm{Sn}$ and ${ }^{58} \mathrm{Ni}$. Here, the photon energy range was $E_{\gamma}=210$ to $220 \mathrm{MeV}$.

Figure 5 shows the integral of the Gaussian corresponding to the coherent events as a function of momentum transfer q after background subtraction. Already here, the typical diffraction pattern due to the $\sin ^{2}\left(\Theta_{\pi}^{*}\right)$ dependence (see eq. 2.3) can be seen. For the determination of the final differential cross section, the photonflux and tagging as well as detection efficiencies have to be taken into account.

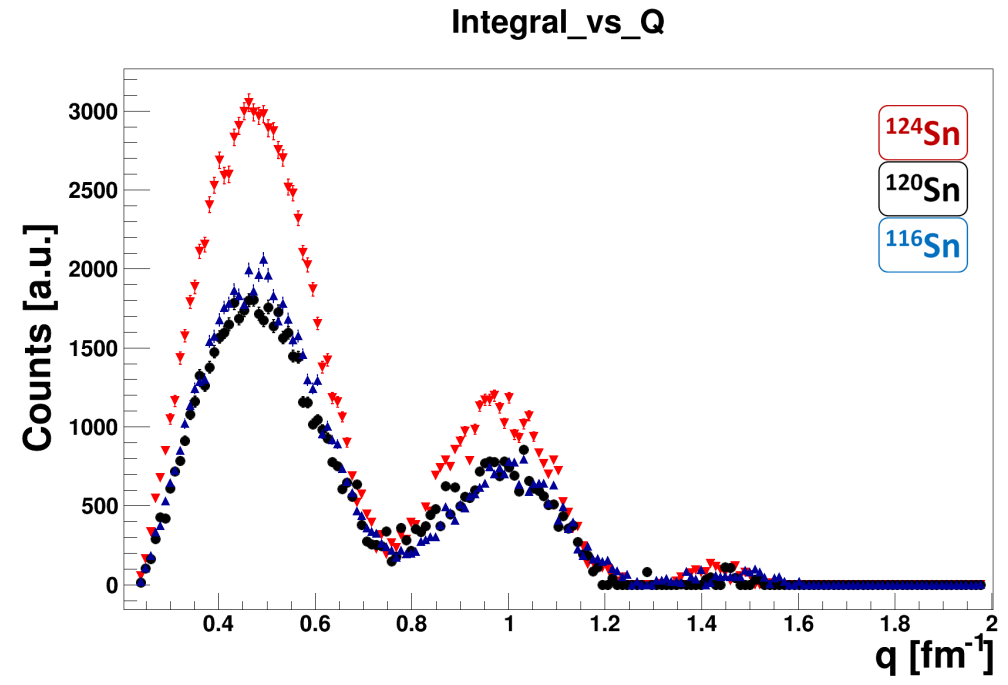

Figure 5: The Integral of the Gaussian representing the coherent events as a function of the momentum transfer $q$. The distribution for ${ }^{124} \mathrm{Sn}$ is shown in red, for ${ }^{120} \mathrm{Sn}$ in black and for ${ }^{116} \mathrm{Sn}$ in blue. The distributions are not yet normalized. 


\section{Summary and Outlook}

The interest on determining the neutron skin thickness of heavy elements has increased over the past years. The coherent $\pi^{0}$ photoproduction, has proven to be capable to access this information in a fast and efficient way [18], allowing a short measuring time per target in the order of 140h. This could permit a systematic scan over the periodic table. To test the feasibility of this idea, an experimental campaign was carried out. Three different tin isotopes $\left({ }^{116,120,124} \mathrm{Sn}\right)$, one nickel $\left({ }^{59} \mathrm{Ni}\right)$ and one lead target $\left({ }^{208} \mathrm{~Pb}\right)$, each with a purity higher than $99.5 \%$ were used. So far, the evolution along the isotopic chain is under investigation.

In order to finally obtain the neutron skin thickness for all the targets, the differential cross section has to be determined. Therefore, a Geant 4 simulation is set up to estimate the detection efficiency of the system. In addition, an interpolated fit with the available theory [19] will then give the radius and diffuseness of the matter distribution [18]. Since the charge distribution is known [1,2], the neutron skin thickness can be extracted.

At last, the extracted skin values can be compared to further theoretical predictions, with the focus on the tin isotopic chain. In figure 6 calculations for various $\mathrm{L}$ and $\mathrm{J}$ parameters (see eq. 1.3) [26] are shown together with present available data. New data with reduced uncertainties would substantially help to constrain these parameters.

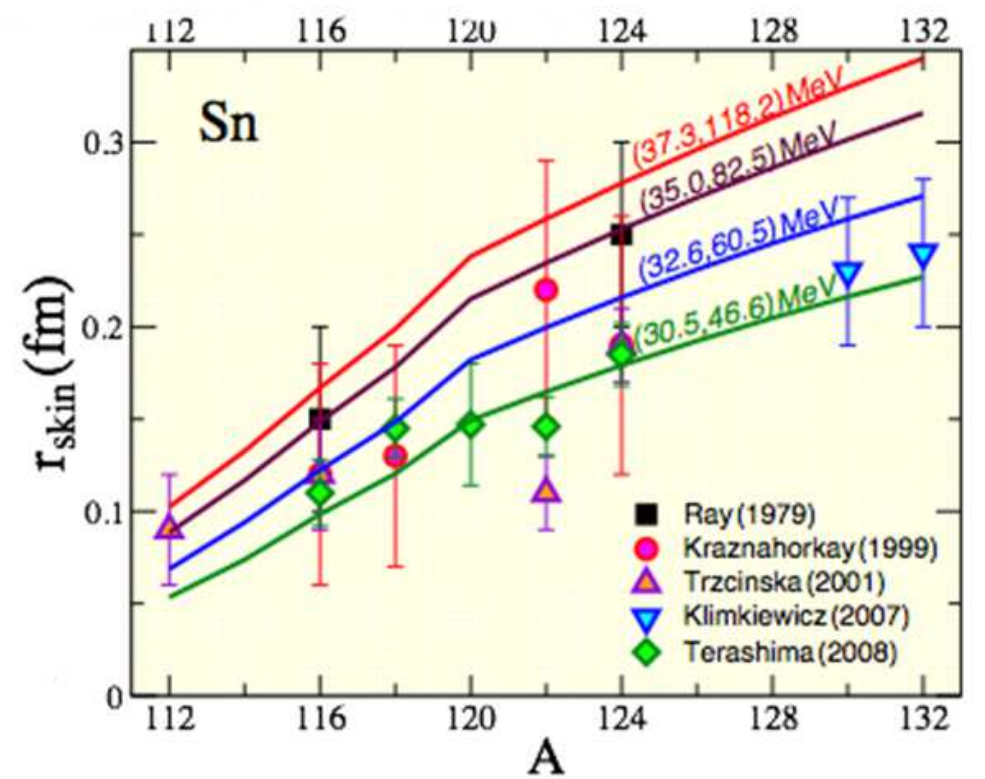

Figure 6: The neutron skin thickness for different tin isotopes as a function of the mass number A. Lines: theoretical prediction with different L and J parameters. Dots: current available data. [27]

\section{Acknowledgments}

This work has been supported by the Deutsche Forschungsgemeinschaft through the Collaborative Research Centre SFB1044. 


\section{References}

[1] B. Frois et al., High-Momuntum-Transfer Electron Scattering from ${ }^{208}$ Pb, Phys. Rev. Lett. 38, 152 (1977)

[2] R. Pohl et al., The size of the proton, Nature 466, 213 (2010)

[3] L. Ray, W. R. Coker, G.W. Hoffmann, Uncertainties in Neutron Densities Determined from Analysis of 0.8 GeV Polarized Proton Scattering from Nuclei, Phys. Rev. C 18, 2641 (1978)

[4] C. Garcia-Recio, J. Nieves and E. Oset, Neutron distributions from pionic atoms, Nuclear Physics A 547, 473 (1992)

[5] A. Trzcinska et al., Neutron Density Distributions Deduced from Antiprotonic Atoms, Phys. Rev. Lett. 87, $082501(2001)$

[6] J. Piekarewicz and S. P. Weppner, Insensitivity of the elastic proton-nucleus reaction to the neutron radius of ${ }^{208} \mathrm{~Pb}$, Nuclear Physics A 778, 10 (2006)

[7] M. B. Tsang et al., Constraints on the symmetry energy and neutron skins from experiments and theory, Phys. Rev. C 86, 015803 (2012)

[8] James M. Lattimer and Yeunhwan Lim, Constraining the Symmetry Parameters of the Nuclear Interaction, The Astrophysical Journal 771, 51 (2013)

[9] M. Warda, X. ViÃ śas, X. Roca-Maza, and M. Centelles, Neutron skin thickness in the droplet model with surface width dependence: Indications of softness of the nuclear symmetry energy, Phys. Rev. C 80, 024316 (2009)

[10] Bao-An Lia, Lie-Wen Chenb, Che Ming Koc, Recent progress and new challenges in isospin physics with heavy-ion reactions, Physics Reports 464, 113 (2008)

[11] B. Alex Brown, Neutron Radii in Nuclei and the Neutron Equation of State, Phys. Rev. Lett. 85, 5296 (2000)

[12] S. Typel and B. Alex Brown, Neutron radii and the neutron equation of state in relativistic models, Phys. Rev. C 64, 027302 (2001)

[13] R.J. Furnstahl, Neutron radii in mean-field models, Nuclear Physics A 706, 85 (2002)

[14] X. Viñas, M. Centelles, X. Roca-Maza, M. Warda, Density dependence of the symmetry energy from neutron skin thickness in finite nuclei, Eur. Phys. J A 50: 27, (2014)

[15] R. A. Schrack, J. E. Leiss, and S. Penner, Neutral Meson Photoproduction from Complex Nuclei, Phys. Rev. 127, 1772 (1962)

[16] Y. Maghrbi et al., Coherent photoproduction of $\pi^{0}$ - and $\eta$-mesons off ${ }^{7}$ Li, The European Physical Journal A 49, 38 (2013)

[17] B. Krusche, Nuclear mass form factors from coherent photoproduction of $\pi$ mesons, The European Physical Journal A 26, 7 (2005)

[18] C.M. Tarbert et al. (Crystal Ball at MAMI and A2 Collaboration), Neutron Skin of ${ }^{208}$ Pb from Coherent Pion Photoproduction, Phys. Rev. Lett. 112, 242502 (2014)

[19] D. Drechsel, L. Tiator, S.S. Kamalov, Shin Nan Yang, Medium effects in coherent pion photo- and electroproduction on ${ }^{4} \mathrm{He}$ and ${ }^{1} 2 \mathrm{C}$, Nucl. Phys. A 660, 423 (1999) 
[20] K.H. Kaiser, K. Aulenbacher, O. Chubarov, M. Dehn, H. Euteneuer, F. Hagenbuck, R. Herr, A. Jankowiak, P. Jennewein, H.J. Kreidel et al., The 1.5-GeV harmonic double-sided microtron at Mainz University, Nucl. Instrum. Meth. A 593, 159 (2008)

[21] I. Anthony, J.D. Kellie, S.J. Hall, G.J. Miller, J. Ahrens, Design of a tagged photon spectrometer for use with the Mainz 840-MeV microtron, Nucl. Instrum. Meth. A 301, 230 (1991)

[22] S.J. Hall, G.J. Miller, R. Beck, P. Jennewein, A focal plane system for the 855-MeV tagged photon spectrometer at MAMI-B Hall, Nucl. Instrum. Meth. A 368, 698 (1996)

[23] A. Starostin et al., Measurement of $K \vec{p} \eta \Lambda$ near threshold, Phys. Rev. C 64, 055205 (2001)

[24] R.Novotny, The BaF 2 photon spectrometer TAPS, IEEE Trans. Nucl. Sci. 38, 379 (1991)

[25] A.R.Gabler et al., Response of TAPS to monochromatic photons with energies between 45 and 790 MeV, Nucl. Instrum. Meth. A 346, 168 (1994)

[26] Lie-Wen Chen, Che Ming Ko, Bao-An Li, and Jun Xu, Density slope of the nuclear symmetry energy from the neutron skin thickness of heavy nuclei, Phys. Rev. C 82, 024321 (2010)

[27] J. Piekarewicz, Neutron Skins and Giant Resonances: A Look Forward, ICNT (2013) 